\title{
EDITORIAL
}

\section{VALORACIÓN ECONÓMICA DEL PATRIMONIO NATURAL}

El desarrollo económico, el crecimiento poblacional y la necesidad de mejorar la calidad de vida han ido generando cada vez mayor presión sobre el uso del patrimonio natural afectándolo negativamente, tanto en su stock, sus flujos, como en su calidad, trayendo como consecuencia la alteración de la base productiva de las actividades económicas y la disminución del bienestar de la población que hace uso de ellos.

De acuerdo al Millennium Ecosystem Assessment (2005) a escala global un 63\% de los servicios ecosistémicos ( 15 de los 24 analizados) en que se sustenta el patrimonio natural, se encuentran en serio declive. Esta disminución en la provisión de los servicios ecosistémicos podría tener un severo Impacto en el bienestar de nuestra sociedad, debido a que cerca de un $70 \%$ de la población vive debajo de la línea de pobreza en áreas rurales, las que en su mayor parte son directamente dependiente de los ecosistemas para desarrollar sus actividades. En este contexto, una correcta identificación de los servicios ecosistémicos y de su valor económico contribuye a reducir la tendencia a subvalorar los ecosistemas, con lo que se incentiva el uso racional de estos.(Daily ,et al 2000)

Una de las herramientas para identificar y valorar los servicios de los ecosistemas es la valoración económica, que a través de métodos y técnicas basadas en la teoría económica busca reconocer, cuantificar e, internalizar los beneficios de estos servicios así como los costos de su pérdida, por los impactos y daños ambientales, de manera que estos bienes y servicios, que no tienen referentes de mercados, puedan incluirse en un análisis costo-beneficio para la toma de decisiones respecto a su mejor gestión.

La toma de decisiones sobre el uso de los ecosistemas y de los servicios que proveen a la sociedad- es una tarea inevitable que está directamente relacionada con la definición de valor y en tanto que en cualquier proceso de toma de decisiones, es necesario contar con un criterio que permita decidir sobre cuál es la mejor alternativa para la sociedad la definición sobre "qué es lo mejor" implica un juicio de valor, por ello la teoría económica ha desarrollado una forma de valorar los servicios suministrados por los ecosistemas naturales, esta valoración se basa en las preferencias subjetivas de los individuos que componen la sociedad; así, el análisis económico intenta alcanzar los objetivos sociales (responder a la pregunta sobre "qué es lo mejor") bajo el concepto de eficiencia, para lo cual emplea el análisis costo-beneficio. Es decir, la sociedad decidirá invertir en un proyecto determinado en la medida que los beneficios que reporte sean mayores a los costos.

Pero, ¿cómo podemos saber el "beneficio" que nos proveerá a la sociedad un proyecto de conservación del bosque si es que no tenemos información de mercado, es decir; de los precios de estos servicios (e.g. control de la erosión)? Es en este contexto que la valoración económica del patrimonio natural cobra importancia, ya que permite asignar un valor monetario a estos servicios del ecosistema que no son capturados en los mercados.

A nivel nacional desde hace más de 15 años se han realizado esfuerzos en elaborar estudios de valoración económica de los recursos naturales, servicios de los ecosistemas, de los componentes de la biodiversidad, es decir del patrimonio natural. Muchos de estos fueron realizados por profesionales de disciplinas de las ciencias naturales, entre ellos biólogos, ingenieros ambientales, quienes vieron en la valoración económica una herramienta que podía ayudar a la conservación. Más adelante entraron los ecosistemas.

La mayoría de los primeros estudios realizados a nivel mundial utilizaron el método de valoración contingente, de igual manera en el Perú se siguió esta tendencia y si bien, en el país son un referente histórico, la gran mayoría de ellos no cumplen con todos los requerimientos para servir de guía a los tomadores de decisión, ya que no siempre están claros los objetivos de la valoración, no se dice cómo usar los valores hallados, o no se aplican los métodos rigurosamente, por diversas razones; como la falta de presupuesto, tiempo, experiencia en la aplicación de métodos, escasa Información biofísica necesaria o por problemas más serios como la falta de comprensión de la necesidad de vincular la valoración, al proceso de toma de decisiones económicas.

\author{
Dr. Cs. Noribal Jorge Zegarra Alvarado \\ Director ESPG - UPT \\ Editor
}

